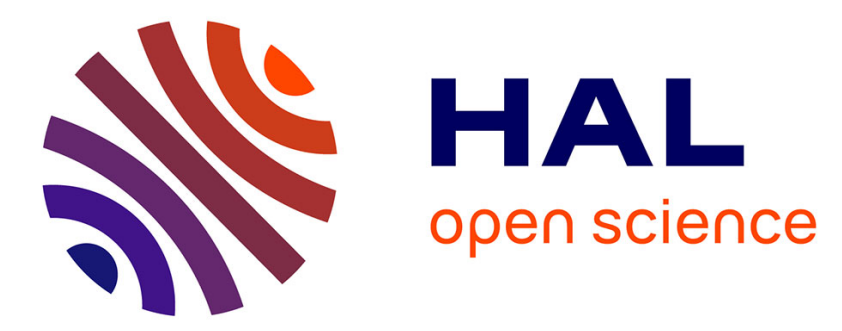

\title{
Protection in Flexible Operating System Architectures
}

Christophe Rippert

\section{To cite this version:}

Christophe Rippert. Protection in Flexible Operating System Architectures. Operating Systems Review, 2003, 37 (4), pp.8-18. 10.1145/958965.958966 . hal-00283925

\section{HAL Id: hal-00283925 \\ https://hal.science/hal-00283925}

Submitted on 1 Jun 2008

HAL is a multi-disciplinary open access archive for the deposit and dissemination of scientific research documents, whether they are published or not. The documents may come from teaching and research institutions in France or abroad, or from public or private research centers.
L'archive ouverte pluridisciplinaire HAL, est destinée au dépôt et à la diffusion de documents scientifiques de niveau recherche, publiés ou non, émanant des établissements d'enseignement et de recherche français ou étrangers, des laboratoires publics ou privés. 


\title{
Protection in Flexible Operating System Architectures
}

\author{
Christophe Rippert \\ Christophe.Rippert@inria.fr \\ SARDES project, LSR-IMAG laboratory \\ INRIA Rhône-Alpes, 655 avenue de l'Europe \\ Montbonnot 38334 St Ismier Cedex, France
}

\begin{abstract}
This paper presents our work concerning flexibility and protection in operating system kernels. In most existing operating systems, security is enforced at the price of flexibility by imposing protection models on the system programmer when building his system. We prove that flexibility can be preserved by separating the management of the protection policy from the tools used to enforce it. We present the secure software framework we have implemented in the THINK architecture to manage protection policies and guarantee they are carried out as specified. We then detail the elementary protection tools provided to the programmer so he can protect his system against unauthorized accesses and denial of service attacks. These tools are implemented in a policy-neutral way so as to guarantee their flexibility. Finally we validate our results by evaluating the flexibility of the protection provided on selected examples of dynamic modification of the protection policy.
\end{abstract}

\section{Introduction}

Flexibility and security are often seen as two conflicting goals in traditional operating systems. Securing a system often results in imposing constraints on the system architecture and on the services provided. On the other hand, improving the flexibility of the system is generally seen as introducing vulnerabilities. For example, recent Linux kernels supports the use of modules, that is system services which can be loaded and unloaded at will in the kernel. This permits the system administrator to add functionnalities to the kernel without having to recompile it, thus enhancing flexibility. However, a malicious user might trick the system administrator into loading a harmful module in the kernel to gain extra privileges for example. On the other hand, forbidding the use of modules strengthens the security of the system but reduces its flexibility since the system administrator is forced to recompile it each time he wants to add a service.

We believe that it is possible to guarantee both flexibility and security in an operating system. Our work focuses especially on protection as defined in [5], that is access control and protection against denial of service attacks. We prove in this paper that flexibility can be preserved in a secure system by separating the management of the protection policy from the tools used to enforce it. The protection policy is the set of rules specifying the access rights of principals and the management of ressources in the system. We thus propose a secure software framework that regulates the interactions between the components in the system and includes the security manager, a component dedicated to the management of policies. Coupled with a set of policy-neutral protection tools, we guarantee that protection policies can be changed dynamically to suit the needs of the system administrator, without needing to recompile or reboot the system, thus preserving its flexibility. Our work is validated using THINK, a flexible system architecture which permits the programmer to build customized operating systems. 
We first present the THINK architecture and the secure software framework we have implemented with it. We then describe the elementary protection tools we have specified and implemented to be policy-neutral. Finally, we present the experiments we have conducted to test the flexibility of our protections tools by dynamically changing the protection policy. We conclude by presenting the future work we plan to conduct concerning the specification of protection policies.

\section{The Think architecture}

The THINK ${ }^{1}$ architecture aims at providing a programming model and a set a tools to ease the task of system builders. This architecture is specified in [4] where its first implementation is also described. This implementation does not address protection issues and let the system vulnerable to abuses. We present below our secure implementation of the THINK software framework, before detailling in Section 3 the elementary protection tools we have devised.

\subsection{Architecture}

THINK is composed of three main entities: the nano-kernel, the service library, and the software framework.

The nano-kernel is the part of the kernel in charge of booting the system and providing access to the underlying hardware. It does not add any system abstraction nor provides any service. It is meant to be as small as possible since it is a mandatory part of the system and we want to minimize the code imposed to the system programmer, as advocated by the Exokernel philosophy [3].

The service library is composed of system services which can be used by the system programmer when he builds his system. Each service is implemented as a stand-alone component. It means that a service can depend on another service's interface (as for example a file system depends on the interface of the hard drive driver), but not on the implementation of this service. Thus, any service can be replaced by another one exporting the same interface. This provides a complete flexibility since no component is mandatory in the system and the programmer can replace any library component by his own if it better suits his needs.

The software framework materializes the programming model advocated in THINK. It is based on the ODP model [6], and includes the well-known notions of interface, component, binding, binding factory, name and naming context. This model specifies the way components must interact, as presented here with the example of a component Src calling the method meth of the component Dst:

1. Component Dst exports its interface with the export method of the naming context interface.

2. Component Src uses the bind method of the binding factory interface to create a binding with the interface exported by component Dst.

3. Component Src calls the method meth of the Dst component using the call method provided by the software framework. In the first implementation of the THINk framework, this call method is in fact a wrapper so as to optimize its performances. It is important to note that methods exported by components cannot be called directly since they are protected by the static $\mathrm{C}$ statement.

\subsection{Implementation}

We present here the secure version of the Think software framework. This framework guarantees that the interactions between the components respect the protection policy specified by the system programmer. We present the implementation of the main notions of the programming model proposed in the THINK architecture.

\footnotetext{
${ }^{1}$ THINK stands for THink Is Not a Kernel
} 


\subsubsection{Interfaces}

Interfaces are implemented as a structure composed of the static name of the exporting component, its methods and its public data. The static name is an unique identifier which can be used to statically define the access rights of the component.

\subsubsection{Name}

Names represent interfaces in a given naming context. Our implementation of a name is a structure composed of an identifier used to find the interface corresponding to the name, and the naming context in which the name is defined.

\subsubsection{The security manager}

The security manager is the component dedicated to the management of security policies on the system, which includes protection policies. Its interface is described in Figure 1.

\begin{tabular}{|cc|}
\hline Secret getSecret(Itf itf); & boolean checkBind(char *staticName, \\
& $\begin{array}{c}\text { Secret secret, } \\
\text { Name dstName); }\end{array}$ \\
boolean checkExport (char *staticName, & boolean checkCall (char *staticName, \\
Secret secret, & Secret secret, \\
Itf itf, & Itf dstIt, \\
NamingContext $\mathrm{nc}) ;$ & char *methStaticName);
\end{tabular}

Figure 1: Interface of the security manager

The getSecret method is called when a component is initialized and returns a secret token. This token permits to dynamically identify a component in a way similar to a PGP public key [9] which identifies a user. In our implementation this token is a 128-bit integer, which makes it statistically very difficult to forge. The checkExport method is used to check that the calling component has the right to export the interface itf. The checkBind method verifies that the calling component can create a binding with the interface dstName. The method checkCall checks that the calling component has the right to call the method methStaticName exported in the interface itf.

\subsection{Naming contexts}

Naming contexts are components managing the naming tables containing the names of the interfaces in the system. The interface of a naming context is presented in Figure 2.

\begin{tabular}{|cc|}
\hline Name byteToName(NamingContext nc, & Name export (NamingContext nc, \\
char *str); & char *staticName, \\
& Secret secret, \\
& Itf itf);
\end{tabular}

Figure 2: The Naming Context interface

The byteToName method takes as a parameter the serialized form of a name and returns that name if it exists in the given naming context. In our implementation, names are serialized as strings. The export method is used by a component identified by its static name and its secret to export an interface in the given 
naming context. It returns a name which identifies the interface in the naming context. This method first calls the checkExport method of the security manager to verify that the component has the right to export the given interface in this naming context.

\subsection{Bindings and binding factories}

Bindings are not represented as components in THINK but they can be seen as communication channels between components. Binding factories are components exporting the interface presented in Figure 3.

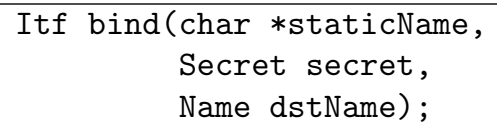

Figure 3: The Binding Factory interface

The bind method creates a binding between the calling component, identified by its static name and secret, and the interface which name is given as a parameter. It returns the interface corresponding to the given name. Before creating the binding, the bind method calls the checkBind method of the security manager to verify that the component has the right to create it.

\subsection{Inter-component calls}

Components call interfaces using the method exported by the inter-component calls component which interface is presented in Figure 4.

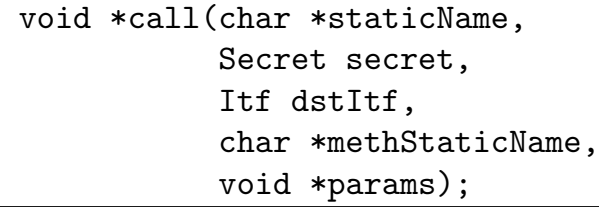

Figure 4: The inter-component calls interface

The call method is used by a component identified by its static name and secret to call a method exported by a given interface. The called method is identified by its static name so as to permit the static specification of call rights. This method first calls the checkCall method of the security manager to verify that the component has the right to call the method. If this verification succeeds, the method is called with the proper parameters.

\section{$3 \quad$ Elementary protection tools}

We have implemented some elementary protection tools that we have included in THINK library. We call these tools elementary since each one can protect the system against a particular threat. All these tools are implemented as components and thus remain independant from each other. We show that these tools are policy neutral which ensures their flexibility. 


\subsection{An adaptable disk manager}

\subsubsection{Principle}

Classical disk managers typically use a geographical scheduling policy which treat request according to the distance between the desired sector and the current position of the head [8]. These algorithms are well suited for all-purpose operating systems but not for specific uses as in real time or multimedia systems for example. Moreover, they are usually vulnerable to denial of service attacks as a malicious process issuing lots of requests so as to slow down the treatment of other requests. We have implemented an adaptable disk manager which permits to dynamically change the scheduling policy. The scheduling algorithm can thus be changed according to the needs of the applications or to counter a denial of service attack.

\subsubsection{Implementation}

The disk manager we have implemented is detailled in Figure 5.

\begin{tabular}{|c|c|c|}
\hline $\begin{array}{l}\text { typedef struct }\{ \\
\text { int readOrWrite; } \\
\text { unsigned sector; } \\
\text { char } * \text { buffer; } \\
\text { unsigned pid; } \\
\text { \} DiskReq; }\end{array}$ & $\begin{array}{r}\text { void initQueue(); } \\
\text { char *readSector (unsigned sect, } \\
\text { char *buffer, } \\
\text { unsigned pid); } \\
\text { void writeSector (unsigned sect, } \\
\text { char *buffer, } \\
\text { unsigned pid); }\end{array}$ & $\begin{array}{l}\text { DiskReq *getQueue(); } \\
\text { void setSched(void }(* f)()) \text {; } \\
\text { void delReq(DiskReq *req); }\end{array}$ \\
\hline
\end{tabular}

Figure 5: Interface of the adaptable disk manager

The DiskReq structure describes a request emitted by a process identified by its process identifier (pid) to a given sector. The initQueue method initializes the request queue and the readSector and writeSector methods permit components such as a file system to send read and write requests to the disk manager. The flushQueue method treats the requests stored in the queue when called by the process managing the burst mode.

The getQueue method is our first elementary tool. It offers a view of the current state of the request queue to the security manager to help him take decisions according to the protection policy.

The setSched method is our second elementary tool. It permits the security manager to change the scheduling algorithm by passing the corresponding function as a parameter to the setSched method. This function is called by the flushQueue method before beginning to treat the requests, so as to sort them in an order matching the scheduling policy.

The delReq method is our third elementary tool. It can be used by the security manager to remove a request from the queue. This is useful during a denial of service attack to clean the queue of the requests emitted by the malicious process.

All these tools are policy neutral since all decisions are made by the security manager. These tools provide the necessary support for the security manager to enforce the protection policy. For example, the security manager can detect a denial of service attack using the getQueue method and change the scheduling algorithm with the setSched method or erase the offending requests with the delReq method. Criteria defining a denial of service attack and the countermeasures to take in case of attack are specified by the protection policy. 


\subsection{Software-based memory isolation}

\subsubsection{Principle}

Memory isolation is a mandatory mechanism in a multi-user operating system. It is indeed necessary to prevent a malicious or malfunctionning process to read or modify other processes' data. However, most operating systems base memory isolation on hardware mechanisms provided by the processor, like segmentation for example. This approach has several drawbacks. First it requires that the memory management unit of the processor provides an isolation mechanism which is not always true especially on embedded systems. Moreover, hardware isolation usually induces a high overhead for interprocess communication since processes must use services as sockets to circumvent the hardware isolation. Finally, hardware isolation lakes flexibility since the test carried out to validate the access is hard coded in the memory management unit and cannot be changed by the system programmer. Thus, we chose to implement a software-based memory isolation mechanism that allow the programmer to fully customize the access control he wants to enforce in his system.

\subsubsection{Implementation}

The tool we have implemented is inspired from the software-based fault isolation mechanisme presented in [7]. When a process is created, the tool parses the binary code of this process and replaces memory accesses with a branch to a well-known entry point in the security manager. This entry point corresponds to a function which task is to check that the memory access is allowed by the protection policy. Thus all memory accesses are checked dynamically when the process code is executed. The access is executed if it is allowed by the protection policy and the execution of the process continues. If the verification fails, a security exception is thrown so as to notify the operating system.

This tool is very flexible since the access control is enforced by a function programmed by the system builder. Thus he can program it as needed to fit the protection policy he wants to enforce in the system. Moreover, our tool permits to choose which memory access must be checked. The programmer can for example decide to verify only branches and not load and store instructions if it suits his needs.

\subsubsection{Evaluation for segment matching}

The tool we have implemented permits to implement any kind of access control. To evaluate it, we have programmed a segment matching policy in the security manager. This policy simply consists in verifying that the destination address of the memory access belongs to a segment defined by its lower and upper bounds. It is very similar to the technique presented in [7]. We detail its operation on a simple example.

The original code of the process is presented in Figure 6. It is composed of a single PowerPC load instruction which task is to copy in register R 1 the content of the memory word at the address computed by adding 8 to the content of register R2.

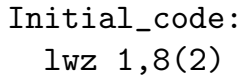

Figure 6: Initial code: 32-bit integer loading

This code is modified by the memory isolation tool by replacing the load instruction by a branch to the checking function in the security manager, as detailled in Figure 7.

The verifying code can be generated dynamically when the process is created since this code is very simple. This permits to generate a code optimized for the replaced instruction, as shown in Figure 8 . For a more complicated verification it may not be possible to dynamically generate the checking function. In our example, the code first calculate the destination address in a temporary register (add 14,2,8), then check this address against the lower and upper bounds, and raise an exception if the address is not in the allowed 
Figure 7: Modified code: branching to the security manager

segment ( $\mathrm{tw} 8,14,15$ and tw $16,14,16$ ). If the test is successful, the original instruction is executed ( $1 \mathrm{wz}$ $1,8(2))$ and the instruction pointer returns to the next instruction in the process (ba Modified_Code + 4).

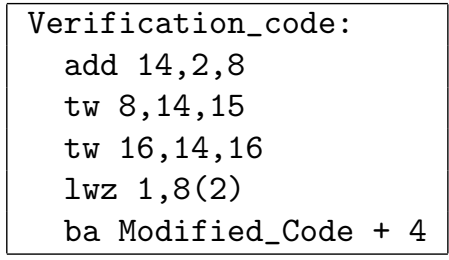

Figure 8: Generated code: checking the destination address and loading the integer if allowed

A simple optimization of this tool is not to generate code for memory access which can be checked before the process execution. For example, the PowerPC platform provides a relative branch instruction for which the destination address is computed by adding an immediate value to the address of the branch instruction. Since this immediate value is coded into the branch instruction itself, the destination address can be computed when the process is created and verified without having to execute the code. This optimization is very effective since relative branches are mostly used in loops for which a dynamic verification for each iteration would be very costly.

\subsubsection{Benchmarks}

Code generation delay The verification code is generated when the process his created in our example for segment matching. We monitored the delay induced by this code generation and found that the process creation delay increases by $3.20 \mathrm{~ms}$ for a test program composed of 29000 instructions (including 9000 memory accesses). Since the time necessary to read the file on the hard drive and allocate the memory for the process is approximately $16.5 \mathrm{~ms}$, the overhead of our tool is only $20 \%$, which seems moderate for most programs.

Execution overhead We monitored the overhead induced by the execution of the verification code for various program types.

Load/store instruction The execution time of a single load or store instruction is multiplied by 3.5 when verified by our tool. This might seem prohibitive but obviously a program is never composed only of load and store instructions.

Relative branch There is no overhead for the exectution of a relative branch since the destination address of this type of instructions can be computed when the process is created, using the optimization presented above.

Absolute branch This type of instructions is mostly used for interprocess communication. We thus compared the cost of an inter-segment call using our software isolation mechanism with the cost of an IPC call over a hardware isolation provided by the processor. The IPC mechanism is based on LRPC [1] and takes $0.490 \mu \mathrm{s}$. With our software isolation tool, the delay is $0.019 \mu \mathrm{s}$ which is 26.4 times faster. Compared to an 
absolute call without any isolation, the overhead is only of $16.67 \%$, which seems very moderate. Softwarebased memory isolation is therefore very interesting for interprocess communication.

Square root computation This algorithm is an example of a program which includes very few memory accesses. It is an iterative algorithm based on Heron of Alexandria's well-known method for computing square roots. The overhead for the computation of the square root of 100000 is only $1.55 \%$ which is obviously negligible.

GZip This program includes lots of memory accesses since it is based on the comparison of strings stored in a dictionnary. Using maximum compression (with the -9 option), the overhead for a $128 \mathrm{MB}$ file is $117 \%$, which can be considered prohibitive.

Conclusion These benchmarks show that performances of a software-based memory isolation mechanism strongly depend of the type of program isolated. However, the system programmer can choose which programs he wants to isolate with it, which is not possible with a hardware isolation. Moreover, the system programmer can customize the access control he wants to enforce by changing the protection policy as needed, thus permitting him to implement lightweight tests for performance critical applications.

\section{Evaluation}

To evaluate the flexibility of our framework and our tools, we present below several situations where the protection policy is dynamically modified during execution of the operating system.

\subsection{Dynamic modification of the memory isolation politic}

\subsubsection{Using the software-based memory isolation tool}

We show on a simple example how our software-based memory isolation tool can be dynamically reconfigurated. In our system, component $\mathrm{C} 2$ is exporting a set of public variables which might be accessed by component $\mathrm{C} 1$, but only after they have been initialized. So the segment containing component $\mathrm{C} 2$ is initially isolated from the segment containing component C1, as shown in the access matrix in Figure 9.

\begin{tabular}{|c|c|c|}
\hline & SegC1 & SegC2 \\
\hline SegC1 & RWX & $\emptyset$ \\
SegC2 & $\emptyset$ & RWX \\
\hline
\end{tabular}

Figure 9: Access matrix for the memory isolation tool

In the security manager, the access rights are stored as capacity lists [5]. These capacities are protected from tampering since the security manager itself is located in a isolated segment. The read, write and execution access rights are stored as boolean which can be modified using the interface presented in Figure 10.

The method getRights permits to get the current access rights of the segment containing the component identified by its static name and secret. This method returns the read, write and execution rights for the segment segSrc. The method setRights permits to set the access rights in a similar way.

This scheme guarantees the flexibility of the isolation. The memory isolation tool generates code to check that the replaced instruction has the right to access the destination segment, which basically means checking that the corresponding boolean is set to true. Thus, all the programmer of component $\mathrm{C} 2$ needs to do to grant to $\mathrm{C} 1$ the access to $\mathrm{C} 2$ public variables after they have been initialized is to set the boolean using the setRights method. The modification of the policy is immediate and the generated code does not have to be changed. Dynamic modification of the isolation policy is therefore very simple thanks to the separation 


\begin{tabular}{|cc|}
\hline void getRights (char *staticName, & void setRights (char *staticName, \\
Secret secret, & Secret secret, \\
unsigned srcSeg, & unsigned srcSeg, \\
boolean *read, & boolean read, \\
boolean *write, & boolean write, \\
boolean *exec); & boolean exec);
\end{tabular}

Figure 10: The access rights modification interface

between the management of the protection policy in the security manager and the tool used to enforce it, that is the software-based memory isolation mecanism.

\subsubsection{Combining the tool and the framework}

The memory isolation tool provides a way to isolate areas of memory from each other. A finer grain access control can be provided by combining the isolation tool and the secure framework implemented in THINK. Figure 11 illustrates an example of a component $\mathrm{C} 1$ calling method meth of component $\mathrm{C} 2$.

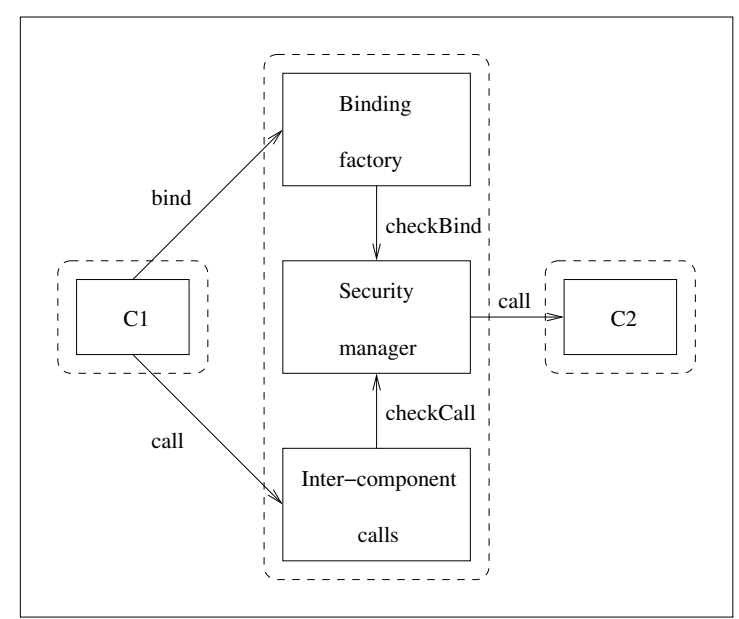

Figure 11: Fine-grain access control between two components

The dashed lines represent segments enforced by the memory isolation tool. The access rights for this isolation are summed up in Figure 12.

\begin{tabular}{|c|c|c|c|}
\hline & SegC1 & SegC2 & SegFramework \\
\hline SegC1 & RWX & $\emptyset$ & $\mathrm{X}$ \\
SegC2 & $\emptyset$ & RWX & X \\
SegFramework & RWX & RWX & RWX \\
\hline
\end{tabular}

Figure 12: Access matrix for the three segments

As can be seen in Figure 12, component $\mathrm{C} 1$ and $\mathrm{C} 2$ can call methods of the framework since it is located in a segment for which they have the execution right, but cannot call each other's methods directly. So component $\mathrm{C} 1$ is forced to use the binding factory and inter-component calls interface to call component 
C2's method meth, thus allowing the framework to check that the protection policy allows that call, using the checkBind and checkCall methods of the security manager.

\subsection{Dynamic modification of the disk scheduling algorithm}

To test the flexibility of our adaptable disk manager, we devised a simple scenario of a denial of service attack. A malicious process which wants to slow down accesses to the disk simply floods the disk manager with requests chosen according to the current scheduling algorithm. For example, if the current algorithm implements a Shortest Seek Time First scheduling [8], the malicious process sends lots of requests for sectors close to the current position of the head so as to cause a starvation for the other processes.

The security manager can detect this kind of attacks using the getQueue method of our adaptable disk manager. A denial of service attack is usually caracterized by lots of requests coming from the same process. The security manager decides according to the protection policy if the current distribution of requests in the queue corresponds to an attack and what countermeasures should be taken. The security must describe the characteristics of an attack since a great number of requests coming from the same process in the queue can be legitimate if this process needs to access a big file on the disk for example.

The protection policy can specify that if a denial of service attack is detected, the current disk scheduling algorithm must be replaced by a fair scheduling algorithm. This type of algorithms reorganizes the requests in the queue according to the process which emitted them. Figure 13 describes an example of this reorganization for two processes P1 and P2 and compares it with a Shortest Seek Time First scheduling. A PxRy request means "request emitted by process Px to read sector y".

\begin{tabular}{|lll|}
\hline Emission order : & [P1R1, P1R8, P2R4, P2R1] \\
Fair scheduling: & [P1R1, P2R4, P1R8, P2R1] \\
SSTF scheduling: & [P1R1, P2R1, P2R4, P1R8]
\end{tabular}

Figure 13: Reorganization of requests by a fair algorithm

It is important to note that this fair algorithm is not meant to be used as a default scheduling algorithm since its performances are much worst than most geographical algorithms. Its goal is only to prevent starvation by interleaving requests from the processes accessing the disk. When using this algorithm, performances will therefore be slowed down equally for all processes, instead of being very high for the attacking process and very low for all the others.

Once the risk of starvation is cancelled by the fair scheduling algorithm, the security manager can use the delReq method to delete from the queue requests coming from the attacking process. It can also ask the process scheduler to kill the offending process if the protection policy requires it. The security manager may need to analyze the request queue for some time before deciding to kill the process, which emphasize the need for a fair disk scheduling algorithm to protect from starvation the processes accessing the disk legitimately during the time needed to take the decision.

This example illustrates the flexibility of the adaptable disk manager. By separating the management of the scheduling policy (implemented by the function passed as a parameter of the setSched method) from the tool used to enforce it (which is the adaptable disk manager itself, with its methods readSector, writeSector and flushQueue), we guarantee that the scheduling policy can be changed dynamically without modifying the tool itself. We chose here an example of a denial of service attack to illustrate a situation where the scheduling algorithm needs to be changed, but the adaptable disk manager can also be useful to adapt the scheduling policy for different types of applications. For example, the system administrator of a general-purpose operating system may want to change the scheduling policy to an algorithm optimized for multimedia applications when such an application is executed on the system, then switch back to a classical geographic algorithm when it is over. 


\section{Conclusion and future work}

As we have seen in this paper, it is possible to ensure both flexibility and protection in an operating system. By separating the management of the protection policy from the tools used to enforce it, we guarantee that the protection policy can be changed dynamically without modifying the tool. The secure framework we have implemented in the THINK architecture includes the security manager, a component dedicated to the management of protection policies. Its interface includes methods ensuring that component interactions are secured and that no component can bypass the framework to escape the protection policy. The elementary protection tools included in THINK library provides the system programmer with support to build a system resistant to unauthorized accesses and denial of service attacks. These tools are programmed to be policyneutral which makes them completely flexible and reconfigurable at will. We believe our results can be applied to any system architecture based on a modular programming model implemented as a flexible software framework.

Some work remains to be conducted concerning the specification of protection policies. In our examples, policies are hard-coded in the security manager, which complicate their dynamic modification. It would be much easier to add and modify policies by using a constraint-based language like Prolog [2]. However, integrating a Prolog interpreter in an operating system is not feasible due to the performance costs it would induce. It would therefore be interesting to specify a constraint-based language which would ease the specification of protection policies without hindering the execution of the system.

\section{References}

[1] B. Bershad, T. Anderson, E. Lazowska, and H. Levy. Lightweight Remote Procedure Call. In Proceedings of the 12th ACM Symposium on Operating Systems Principles, 1989.

[2] P. Deransart, A. Ed-Dbali, and L. Cervoni. Prolog: The Standard. Springer, 1996.

[3] D. R. Engler, M. F. Kasshoek, and J. O'Toole. Exokernel: An Operating System Architecture for Application-Level Resource Management. In Proceedings of the 15th ACM Symposium on Operating Systems Principles, 1995.

[4] J-Ph. Fassino, J-B. Stefani, J. Lawall, and G. Muller. THINK: A Software Framework for Componentbased Operating System Kernels. In Proceedings of the Usenix Annual Technical Conference, 2002.

[5] B. W. Lampson. Protection. In Proceedings of the 5th Princeton Conference on Information Sciences and Systems, 1971.

[6] ODP Reference Model, Foundations, ITU-T ISO/IEC Recommendation X.902 International Standard 10746-2, 1995.

[7] R. Wahbe, S. Lucco, T. E. Anderson, and S. L. Graham. Efficient Software-Based Fault Isolation. ACM SIGOPS Operating Systems Review, 27(5):203-216, 1993.

[8] B. L. Worthington, G. R. Ganger, and Y. N. Patt. Scheduling Algorithms for Modern Disk Drives. In Proceedings of the 1994 ACM SIGMETRICS conference on Measurement and Modeling of Computer Systems, 1994.

[9] P. R. Zimmermann. PGP: Source Code and Internals. MIT Press, 1995. 